\title{
Twisted Eguchi-Kawai Reduced Chiral Models
}

\author{
Stefano Profumo \\ Scuola Internazionale Superiore di Studi Avanzati \\ Via Beirut 2-4, I-34014 Trieste, Italy \\ E-mail: profumo@sissa.it \\ Ettore Vicari \\ Dipartimento di Fisica dell'Università di Pisa and I.N.F.N. \\ Via Torricelli 2, I-56127 Pisa, Italy \\ E-mail: vicari@df.unipi.it
}

\begin{abstract}
We study the twisted Eguchi-Kawai (TEK) reduction procedure for large $N$ unitary matrix lattice models. In particular, we consider the case of twodimensional principal chiral models, and use numerical Monte Carlo (MC) simulations to check the conjectured equivalence of TEK reduced model and standard lattice model in the large- $N$ limit. The MC results are compared with the large- $N$ limit of lattice principal chiral models to verify the supposed equivalence. The consistency of the TEK reduction procedure is verified in the strong-coupling region, i.e. for $\beta<\beta_{c}$ where $\beta_{c}$ is the location of the large- $N$ phase transition. On the other hand, in the weak-coupling regime $\beta>\beta_{c}$, relevant for the continuum limit, our MC results do not support the equivalence of the large- $N$ limits of the lattice chiral model and the corresponding TEK reduction. The implications for the correspondence between TEK model and noncommutative field theory are also discussed.
\end{abstract}

Keywords: Matrix Theories, 1/N Expansion, Lattice Quantum Field Theories, Lattice Gauge Field Theories. 


\section{Contents}

1. Introduction 1

2. TEK reduced chiral models 2

$2.1 \quad$ TEK reduced models 2

2.2 Principal chiral models 1

2.3 The reduced version: symmetries and equivalence

3. Numerical results 6

4. Conclusions 10

\section{Introduction}

A renewed interest in reduced matrix models has been motivated by the recent discovery of their relevance to noncommutative field theories and to superstring matrix models, see, e.g., Refs. [1, 2, 3, 4, 5, 6, 7, 8, 9] and references therein. Reduced matrix models were introduced for the study of the large- $N$ limit of $S U(N)$ gauge theories, see, e.g., Ref. [10] for a review. Eguchi and Kawai [11] pointed out that, as a consequence of the large- $N$ factorization, one can construct one-point theories equivalent to lattice $S U(N)$ gauge theories in the limit $N \rightarrow \infty$. The original proposal was just the replacement of all link variables $U_{\mu}(x)$ of the Wilson formulation of lattice gauge theories with four $S U(N)$ matrices according to the reduction rule $U_{\mu}(x) \rightarrow U_{\mu}$. This simple reduction procedure was shown to fail in the weak-coupling region, because of the spontaneous breaking of a symmetry that is crucial for the equivalence [12]. Modifications of the original Eguchi-Kawai model have been then proposed [12, 13, 14]. The most promising one is represented by the so-called twisted Eguchi-Kawai (TEK) reduction [13, 15], which replaces $U_{\mu}(x) \rightarrow D(x) U_{\mu} D(x)^{\dagger}$, where $D(x)=\prod_{\mu} \Gamma_{\mu}^{x_{\mu}}$ and $\Gamma_{\mu}$ are appropriate $S U(N)$ matrices obeying the 't Hooft algebra.

Since the proposal of the application of the TEK reduction prescription [13, 15], the numerical checks [14, 16, 17, 18] of the validity of the procedure have not been, to our opinion, completely exhaustive. We believe that, also in view of the renewed interest for matrix models, and particularly for TEK reduced versions, it is important to perform an accurate test of the conjectured equivalence with the corresponding lattice matrix model. This point may eventually be of relevance for the rôle of TEK 
models in noncommutative field theories or in superstring matrix (toy) models, where the theory should be analyzed in a limit different from the planar one.

In this paper we study the application of the twisted Eguchi-Kawai (TEK) reduction procedure to two-dimensional principal chiral models on the lattice [13, 19, 20, 21]. Two-dimensional chiral models and four-dimensional gauge theories present interesting analogies, see, e.g., Refs. 222, 23, 24, 25]. For example, they are both asymptotically free unitary matrix theories, and their large- $N$ limit is represented by a formal sum of planar graphs. In the large $N$ limit, one can apply the TEK reduction procedure to both lattice theories. In both cases the validity of the prescription is demonstrated by showing that the Schwinger-Dyson equations for the reduced and the non-reduced theory become formally the same in the limit $N=\infty$. In two-dimensional lattice chiral models a stringent test of the TEK reduction procedure should be simplified, for essentially three reasons: a better knowledge of the large- $N$ limit of the lattice chiral model, an easier way to define observables in spin models, and a simpler reduced model constituted by only one unitary matrix.

It has been recently realized that TEK reduced model may provide a nonperturbative formulation of noncommutative field theories 田, 5, 6, 8, 9. In particular, one may establish a correspondence between the TEK reduced model of the lattice

principal chiral theory and a noncommutative $\mathrm{U}(1)$ principal chiral model [26]. This is formally achieved in a continuum limit that keeps the noncommutativity parameter $\theta_{\mu \nu}$ fixed 4 , 5, 6, 8, 9, where $\theta_{\mu \nu}$ scales as $a^{2} N$ and $a$ is the lattice spacing. Assuming the equivalence of the TEK reduced and the commutative principal chiral model in the planar limit, one may rewrite this condition as a double scaling limit involving the lattice coupling and $N$ [26]. Therefore, our study of TEK reduced model in the planar limit is also useful to clarify the correspondence between TEK model and noncommutative field theory, and therefore the possibility of achieving a nonperturbative lattice formulation of a noncommutative field theory.

The plan of the paper is as follows. In Sec. 2 we introduce the original field theory and its reduced counterpart, and show on what grounds one expects that it coincides with the non-reduced theory in the large $N$ limit. In Sec. 3 we present our numerical results obtained by performing Monte Carlo simulations. Finally, Sec. 1 contains comments and conclusions.

\section{TEK reduced chiral models}

\subsection{TEK reduced models}

Twisted Eguchi-Kawai reduced models are based on the idea that, when $N \rightarrow \infty$, the $\mathrm{SU}(N)$ group becomes so large that it may accommodate the full Poincarè group as a subgroup, and allow us to find representations of the translation and rotation operators among the elements of $\mathrm{SU}(N)$. As a consequence, one may reformulate 
the full theory in terms of a finite number of matrix field variables defined at a single space-time site (or on the $d$ links emerging from the site in the case of a lattice gauge theory) and of the above-mentioned representations of the translation group. This reformulation is called "twisted Eguchi-Kawai" reduced version of the theory [13, 15].

Consider a lattice field theory with fields $\phi(x)$ in the adjoint representation of $S U(N)$. The twisted reduction prescription reads:

$$
\phi(x) \rightarrow D(x) \phi D^{\dagger}(x),
$$

where

$$
D(x)=\prod_{\mu}\left(\Gamma_{\mu}\right)^{x_{\mu}},
$$

and $\Gamma_{\mu}$ are $S U(N)$ matrices obeying the so called t'Hooft algebra:

$$
\Gamma_{\mu} \Gamma_{\nu}=Z_{\mu \nu} \Gamma_{\nu} \Gamma_{\mu},
$$

where $Z_{\mu \nu}$ is an element of the center of the group, $\mathbb{Z}_{N}$,

$$
Z_{\mu \nu}=\exp \left(\frac{2 \pi i}{N} n_{\mu \nu}\right)
$$

and $n_{\mu \nu}$ is an integer valued antisymmetric $d \times d$ matrix (in $d$ dimensions). $\Gamma_{\mu}$ is the matrix implementing the translation by one lattice spacing in the $\mu$ direction. The reduced action is simply obtained by substituing (2.1) into the action of the original lattice field theory:

$$
S_{T E K}\left(\phi, n_{\mu \nu}\right)=\frac{1}{\operatorname{vol}} S\left[D(x) \phi D^{\dagger}(x)\right],
$$

and the partition function reads:

$$
Z_{T E K}=\int \mathcal{D} \phi \exp \left(-S_{T E K}\right),
$$

for a fixed value of $Z_{\mu \nu}$. The expectation value of any functional of the reduced field $\phi$ is given by:

$$
\langle O(\phi)\rangle_{T E K}=\frac{1}{Z_{T E K}} \int \mathcal{D} \phi O(\phi) \exp \left(-S_{T E K}\right)
$$

The correspondence between correlation functions of the reduced model and of the original field theory is as follows. Let $f(x)$ be any invariant functional of the field $\phi(x)$. Then

$$
\langle f[\phi(x)]\rangle_{\text {field theory }}=\left\langle f\left[D(x) \phi D^{\dagger}(x)\right]\right\rangle_{T E K}
$$

The actual check of validity of the reduction procedure is based on the comparison of the Schwinger-Dyson equations of the reduced and original models. This procedure however requires some attention, since the limit of infinitely many degrees of freedom within the group itself allows the possibility of spontaneous breakdown of some of the symmetries which would be preserved for any finite value of $N$. Moreover, large $N$ is a thermodynamical limit: $N$ must go to infinity before any other limit is considered, and sometimes the limiting procedures do not commute. 


\subsection{Principal chiral models}

The two-dimensional principal chiral field theory is defined through the following action

$$
S=\frac{1}{T} \int d^{2} x \operatorname{Tr}\left[\partial_{\mu} U(x) \partial_{\mu} U^{\dagger}(x)\right],
$$

where $U(x)$ are unitary matrix variables. The action is naturally invariant under the transformations $U \rightarrow V_{L} U$ and $U \rightarrow U V_{R}$ where $V_{L}, V_{R} \in U(N)$. As amply discussed in the literature (see, e.g., Refs. [23, 24, 25] and references therein), two-dimensional chiral models and four-dimensional lattice gauge theories manifest deep analogies, such asymptotic freedom, large- $N$ planar limit, etc...

A corresponding lattice principal chiral model can be obtained by the usual substitution of the derivative with a finite difference, obtaining

$$
S=-\beta N \sum_{x} \sum_{\mu=1,2} \operatorname{Tr}\left[U_{x} U_{x+\mu}^{\dagger}+U_{x+\mu} U_{x}^{\dagger}\right],
$$

where $\beta N \equiv 1 / T$. In view of a large- $N$ analysis one may consider both $S U(N)$ and $U(N)$ models, since they are expected to reproduce the same statistical theory in the limit $N \rightarrow \infty$, which is meant at fixed $\beta$. Moreover, $S U(N)$ and $U(N)$ chiral models have the same continuum limit at any finite $N \geq 2$.

For a recent review of results concerning chiral models see, e.g., Ref. [24]. Here we only mention the presence of a peculiar large- $N$ phase transition [27, 28, 29, 30] which manifests itself with a power-law singularity in the $N \rightarrow \infty$ limit of the specific heat, i.e. $C_{\infty} \sim\left|\beta_{c}-\beta\right|^{-\alpha}$, and with a peak in the specific-heat at finite $N$ that becomes sharper and sharper with increasing $N$ [28, 30]. The analysis of the $N=\infty$ strongcoupling series [29] provides the estimates $\beta_{c}=0.3060(4)$ and $\alpha=0.27(3)$, while the extrapolations to $N=\infty$ of the position of the specific-heat peak, determined by Monte Carlo simulations for $S U(N)$ and $U(N)$, gives $\beta_{c}=0.3957(3)$.

\subsection{The reduced version: symmetries and equivalence}

Implementing (2.1) into (2.10) gives the TEK reduced lattice chiral model action:

$$
S_{T E K}=-\beta N \sum_{\mu=1,2} \operatorname{Tr}\left[U \Gamma_{\mu} U^{\dagger} \Gamma_{\mu}^{\dagger}+\text { h.c. }\right] .
$$

In the present case, $n_{\mu \nu}$ of equation (2.4) is generically, in $d=2$, of the form

$$
n_{\mu \nu} \equiv\left(\begin{array}{cc}
0 & M \\
-M & 0
\end{array}\right), \quad M \in \mathbb{Z} .
$$

For a given $N$ and $M$ the solution to ([31) is provided, up to global $S U(N)$ transformations, by the $N \times N$ shift and clock matrices

$$
\begin{gathered}
S_{i, j}^{(M)} \equiv \delta_{i+M, j}, \\
C_{i, j} \equiv e^{\frac{2 \pi i}{N}(i-1)} \delta_{i, j} .
\end{gathered}
$$


The two matrices $\Gamma_{\mu}$ are given respectively by $S$ and $C$.

While the original field theory is invariant under the $S U(N)_{L} \times S U(N)_{R}$ group, the reduced theory is invariant under the following two symmetries:

$$
\begin{aligned}
& U \rightarrow z \cdot U \quad z \in \mathbb{Z}_{N}, \\
& U \rightarrow D(x) U D^{\dagger}(x) .
\end{aligned}
$$

The first symmetry corresponds to the contraction of the original symmetry of the model to the center of the algebra of the symmetry group, while the second one is reminiscent of the translational invariance of the original theory.

In order to show the equivalence of the reduced theory with the original field theory in the large $N$ limit, the starting point is given by the Schwinger-Dyson equations, i.e. the equation obtained from a generic correlation function in the original theory (the reduced case is the obvious translation using Eq. (2.1])

$$
G^{(n)}=\left\langle\operatorname{Tr}\left(\prod_{i=1}^{n} U_{x_{i}}^{k_{i}}\right)\right\rangle,
$$

by the following steps:

1. substitute $U_{x_{i}}^{k_{i}}$ with $\lambda^{a} U_{x_{i}}^{k_{i}}$, where $\lambda^{a}$ is a $S U(N)$ generator;

2. implement the change of variables $U \rightarrow\left(1+i \alpha \lambda^{b}\right) U$ in the integral (implicitly taken in the average), using the Haar measure invariance;

3. contract the $a, b$ indices and extract the linear part in the parameter $\alpha$.

By comparing the two sets of equations obtained in the original and in the reduced theory, one finds manifestly corresponding terms and also some extra terms in the reduced theory. These extra terms exactly cancel if the symmetry $U \rightarrow D(a) U D^{\dagger}(a)$ of the reduced model is not spontaneously broken [19]. Therefore, the two models possess the same Schwinger-Dyson equations if the above mentioned symmetry is not broken in any regime, and in particular in the continuum limit. The point is then whether this implies the equivalence of the two models, i.e. that they have the same expectation values of invariant quantities. Since the large $N$ equivalence of the twisted reduced models is based on the above arguments even for the lattice gauge theory case, it is an important task to verify, if the invoked symmetry holds, whether the Schwinger-Dyson equation based demonstration of the equivalence is indeed conclusive and sufficient or not.

The equivalence of the lattice chiral model and the reduced TEK model in the $N=\infty$ limit is not actually questioned in the strong-coupling region, i.e. for sufficiently small values of $\beta$. In this region the symmetries of the TEK reduced model are unbroken and therefore the equivalence of Schwinger-Dyson equations holds. One 
may then argue that this is sufficient to yield the equivalence of the reduced and nonreduced models. In the strong-coupling domain, i.e. within the convergence radius of the strong-coupling expansion, expectation values are analytic in the coupling $\beta$, and their boundary value at $\beta=0$ can be easily computed. As a consequence, it is formally possible to solve the Schwinger-Dyson equations in terms of strongcoupling series by iterative methods. Since the $N=\infty$ boundary values at $\beta=0$ of the lattice chiral model and the reduced TEK model coincide, equal SchwingerDyson equations imply the formal equivalence within the convergence radius of the strong coupling expansion ${ }^{1}$. Problems may occur in the weak-coupling side of a large- $N$ phase transition.

It has been also argued that [10 the reduced TEK model is, in a sense, equivalent to a theory on a box of size $L=N$. In the large- $N$ limit the finite- $N$ corrections should be $O\left(1 / N^{2}\right)$, just as in the $S U(N)$ lattice chiral theory. Since $N^{2}=L^{2}$, finite$N$ corrections can be seen as finite volume corrections. Therefore, one may expect that the asymptotic large- $N$ regime is reached for $N>>\xi$, where $\xi$ is the $N=\infty$ correlation length at the given value of $\beta$. Note that in TEK reduced models the large- $N$ and thermodynamic limits are connected and approached simultaneously, while in the standard lattice models one first performs the $L \rightarrow \infty$ limit and then the large- $N$ limit. In a general field theory there are no a priori reasons why these two limits should commute.

\section{Numerical results}

In order to study the large- $N$ limit of the TEK model, we have performed Monte Carlo simulations for several values of $N$, up to $N=200$, and for values of $\beta$ in the strong and weak coupling regime. We used a Metropolis algorithm to update the $S U(N)$ matrix $U$. Trial matrices were selected by multiplying the actual matrix $U$ by a random $\mathrm{SU}(2)$ matrix embedded in $S U(N)$, choosing randomly among the $N(N-1) / 2 S U(2)$ subgroups. More precisely, once the $S U(2)$ subgroup is randomly chosen, we performed ten Metropolis hits with an approximate acceptance of $50 \%$. Each $S U(2)$ updating requires $O(N)$ operations. The number of $S U(2)$-subgroup updatings per run was $O\left(10^{9}\right)$. We should also mention that for large values of $N$, $N \gtrsim 60$ say, we observed some problem of thermalization when using completely random configurations (for example, constructed by multiplying $N(N-1) / 2$ completely random $\mathrm{SU}(2)$ matrix embedded in a $N \times N$ and associated to different subgroups) as starting point of our simulations. This occurred in both the strong- and weakcoupling regions, worsening with increasing $\beta$. So, as starting point we used either moderately random matrices or the unity matrix. It is well known that a simple

\footnotetext{
${ }^{1}$ Notice that the convergence radius of the strong coupling expansion does not necessarily coincide with the large- $N$ transition point $\beta_{c}$, but it may be smaller.
} 
Metropolis algorithm does not provide a particularly efficient method to simulate a statistical system. Our choice was essentially due to the fact that the reduced action is quadratic in the matrix variable $U$. Moreover, it does not lend itself to a linearization by introducing new matrix variables, as in the case of the reduced TEK gauge theory [17].

\begin{tabular}{ccc}
\hline \hline$N$ & $E$ & $\chi$ \\
\hline 10 & $0.6155(2)$ & $12.30(2)$ \\
16 & $0.6346(1)$ & $12.47(2)$ \\
20 & $0.6409(1)$ & $11.46(4)$ \\
30 & $0.64766(7)$ & $9.49(3)$ \\
40 & $0.65046(8)$ & $8.44(3)$ \\
50 & $0.65159(5)$ & $7.94(3)$ \\
60 & $0.65226(4)$ & $7.65(3)$ \\
80 & $0.65285(3)$ & $7.34(4)$ \\
100 & $0.65329(4)$ & $7.21(4)$ \\
150 & $0.65349(4)$ & $7.08(5)$ \\
\hline \hline
\end{tabular}

In our simulations we measured the internal energy

$$
E=1-\frac{1}{N}\left\langle\operatorname{Tr} U \Gamma_{\mu} U^{\dagger} \Gamma_{\mu}^{\dagger}\right\rangle,
$$

the quantity corresponding to the two-point function $G(x)=\left\langle\operatorname{Tr} U(0) U(x)^{\dagger}\right\rangle$ using the equivalence (2.8), and in particular its low-momentum components, such as the (zero-momentum) magnetic susceptibility

$$
\chi=|\operatorname{Tr} U|^{2} .
$$

Table 1: Internal energy $E$ and magnetic susceptibility $\chi$ for $\beta=$ 0.28 .

In order to check for possible spontaneous breaking of the symmetries (2.14) and (2.15), we considered the two quantities

$$
\begin{aligned}
& S_{1}=\frac{1}{N}\langle\operatorname{Tr} U\rangle, \\
& S_{2}=\frac{1}{N}\left\langle\operatorname{Tr} U \Gamma_{1} U^{\dagger} \Gamma_{2}^{\dagger}\right\rangle .
\end{aligned}
$$

Indeed, $S_{1}$ is not invariant under the transformation (2.14) (but it is invariant with respect to (2.15)), while $S_{2}$ is not invariant under the transformation (2.15) (but it is invariant with respect to (2.14)).

Information on the large- $N$ limit of lattice chiral models in the strong coupling region can be obtained from the $N=\infty$ strong-coupling expansion and extrapolation of Monte Carlo results for the $S U(N)$ and $U(N)$ unitary groups. $N=\infty$ strongcoupling series are reported in Refs. [22, 27, 28, 29]. For example, the internal energy is known to 17th order and the magnetic susceptibility to 15th order. Monte Carlo simulations for relatively large values of $N$ (up to $N=30$ ) can be found in Refs. [28, 30]. It is useful to consider both $S U(N)$ and $U(N)$ data because their expectation values aproach the $N=\infty$ values from opposite sides, and allow us to have a better control of the large- $N$ limit.

As already mentioned, the equivalence of reduced and nonreduced models is not questioned in the strong-coupling region. However, Monte Carlo simulations in the strong coupling region are still useful to study the convergence of the large- $N$ limit of TEK, which is expected to be controlled by powers of $N^{-2}$. 
After verifying a general agreement between the large- $N$ TEK results and the $N=\infty$ strong-coupling series for small $\beta$, we have performed longer runs for $\beta=0.28$ in order to obtain an accurate test. This value of $\beta$ is not too far from the large- $N$ transition point $\beta_{c} \approx 0.306$. The large- $N$ limit of $E$ and $\chi$ of the lattice chiral model can be estimated from the analysis of the corresponding strong-coupling series [29], obtaining ${ }^{2} E_{\infty}=0.65370(4)$ and $\chi_{\infty}=7.02(1)$. Extrapolations of the Monte Carlo results reported in Refs. [28, 30], using the fact that the corrections are expected to be powers of $N^{-2}$, provide consistent results, i.e. ${ }^{3} \chi_{\infty}=7.00(2)$ for $S U(N)$, $\chi_{\infty}=7.02(2)$ and $E_{\infty}=0.65373(4)$ for $U(N)$. Moreover, we mention that the large$N$ limit of the second-moment correlation length is $\xi_{\infty} \approx 1.53$. In Table 1 we report $E$ and $\chi$ as obtained by our simulations for several values of $N$, up to $N=150$. In Figs. 1 and 2 we plot them versus $1 / N^{2}$, which is the order of the expected asymptotic corrections. There we also show the MC data obtained from simulations of $S U(N)$ and $U(N)$ lattice chiral models. The figures show clearly that the correct $N=\infty$ limit is approached. To be more quantitative, fitting the data for $N \geq 30$ allowing for a $O\left(N^{-2}\right)$ correction, we obtained

$$
\begin{aligned}
& E \approx 0.65377(3)-\frac{5.46(7)}{N^{2}}, \\
& \chi \approx 7.02(2)+\frac{2.24(4) \times 10^{3}}{N^{2}},
\end{aligned}
$$

with acceptable $\chi^{2} /$ d.o.f $\approx 1$. This analysis shows that the correct large- $N$ limit is approached with corrections of $O\left(N^{-2}\right)$, as expected. Note, however, that the $O\left(1 / N^{2}\right)$ corrections are much larger than those found for the lattice chiral models, by about a factor $10^{2}$ for $\chi$, see the footnote 3 .

As already mentioned, lattice unitary chiral models present a peculiar large- $N$ phase transition, which is characterized by a power-law singularity of the specific heat at $N=\infty$ 29. At finite $N$, numerical simulations show that the peak in the specific-heat becomes sharper and sharper with increasing $N$ [30]. A peak in the specific heat is also observed in the TEK reduced model: it becomes sharper and sharper with increasing $N$. The $N \rightarrow \infty$ extrapolation of its location provides the estimate $\beta_{c} \approx 0.306$, in agreement with the results for the lattice chiral models.

These results provide an accurate check that for $\beta<\beta_{c}$ the TEK model shows the expected behavior, i.e. it converges to the same large- $N$ limit of lattice chiral model, with corrections of $O\left(N^{-2}\right)$. We should also observe that, due to the large

\footnotetext{
${ }^{2}$ Following Ref. [29], we used Dlog-Padé approximants to resum the series of $d E / d \beta \sim\left(\beta_{c}-\beta\right)^{-\alpha}$ and $d \ln \chi / d \beta \sim\left(\beta_{c}-\beta\right)^{-\alpha}$ with $\beta_{c} \approx 0.306$ and $\alpha \approx 0.27$. The internal energy and the magnetic suceptibility are then obtained by integrating with respect to $\beta$.

${ }^{3}$ For example, the simplest extrapolation using a straight line $a+b N^{-2}$ passing by the data for the largest values of $N$ (i.e. $\chi(N=30)=7.032(5), \chi(N=21)=7.069(5)$ for $S U(N)$ and $\chi(N=21)=6.972(8), \chi(N=15)=6.924(7)$ for $U(N))$ gives $\chi(N)=7.00(1)+32(6) N^{-2}$ for $S U(N)$ and $\chi(N)=7.02(2)-22(5) N^{-2}$ for $U(N)$.
} 


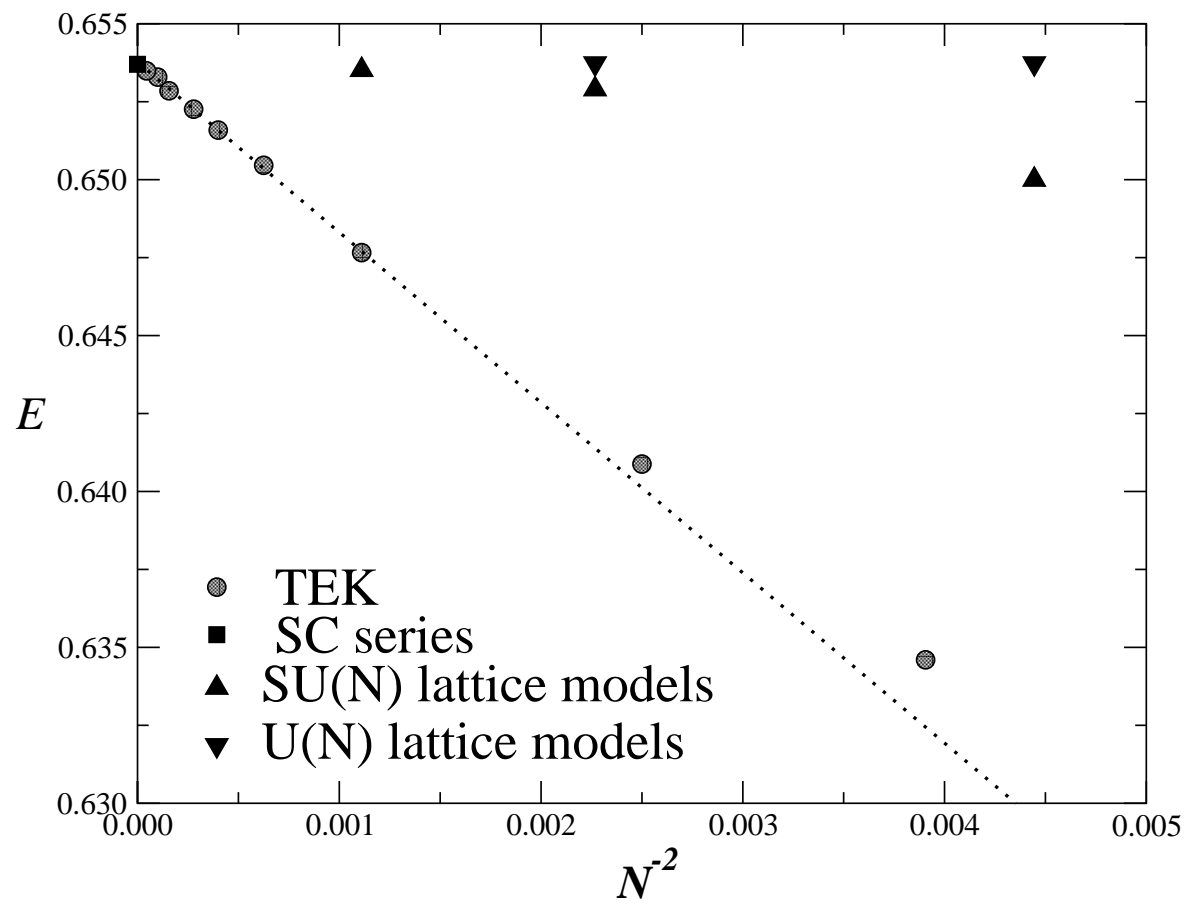

Figure 1: The internal energy versus $N^{-2}$ for $\beta=0.28$. We also show the large- $N$ limit $E_{\infty}$ as obtained from the analysis of the $N=\infty$ strong-coupling series, and results from simulations of $S U(N)$ and $U(N)$ lattice chiral models.

corrections, the convergence to the $N=\infty$ limit is rather slow, much slower than the convergence of the lattice chiral models. Comparing with the numerical effort to reach a similar accuracy in simulations of lattice chiral models, the reduced TEK model does not appear to be convenient to numerically investigate the large- $N$ limit of chiral models, at least in the strong-coupling domain.

Let us now turn to the weak-coupling region, i.e. for $\beta>\beta_{c}$. In this case information on the large- $N$ limit of lattice chiral models comes only from the $\mathrm{MC}$ simulations of Refs. [28, 30]. We performed runs for $\beta=0.31$ and $\beta=0.32$, for which the $N=\infty$ correlation length are estimated to be $\xi \approx 4$ and $\xi \approx 6$, respectively. MC simulations up to $N=200$ for these values of $\beta$ should provide a convincing check of the large- $N$ convergence, which should be controlled by the ratio $N / \xi$ according to the arguments mentioned in Sec. 2.3. The results of the simulations for $E, \chi$, and the two quantities $S_{1}$ and $S_{2}$, cf. Eqs. (3.3) and (3.4), are reported in Table 2 . They should be compared with the the extrapolations to $N \rightarrow \infty$ of the MC results reported in Ref. [28, 30], that are $\chi_{\infty}=34.1(2)$ and $E_{\infty} \approx 0.519$ for $\beta=0.31$, and $\chi_{\infty} \approx 65$ and $E_{\infty} \approx 0.485$ for $\beta=0.32$. Our MC data are also plotted in Figs. 3 and \#, where we also show the MC results for $S U(N)$ and $U(N)$ lattice chiral models, taken from Refs. [28, 30]. While the data for the energy seem somehow to approach the corresponding $E_{\infty}$ (although it is not evident that the corrections are $O\left(N^{-2}\right)$ ), those for $\chi$ do not show any evidence of convergence to $\chi_{\infty} \cdot \chi$ appears to increase 


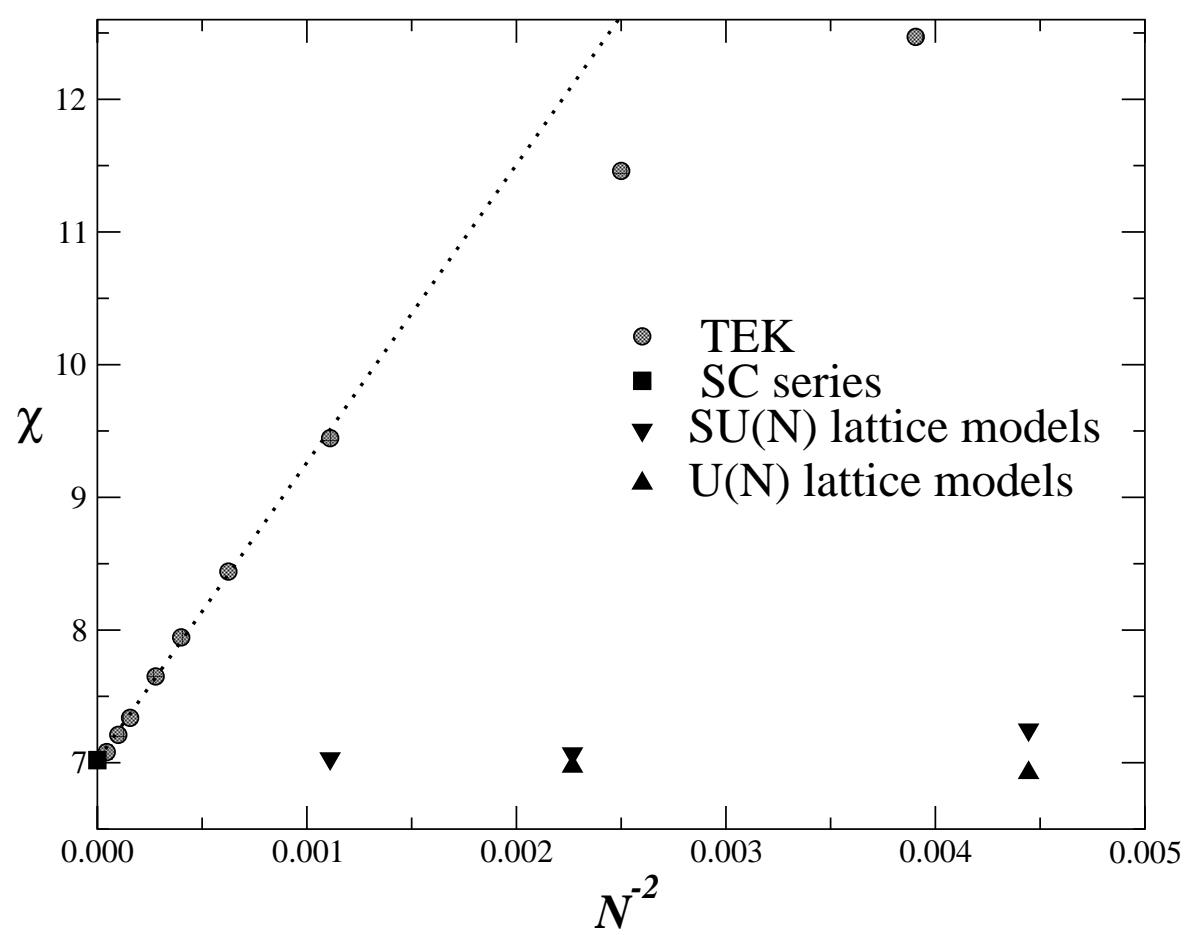

Figure 2: The magnetic susceptibility versus $N^{-2}$ for $\beta=0.28$. We also show the large- $N$ limit $\chi_{\infty}$ as obtained from the analysis of the $N=\infty$ strong-coupling series, and results from simulations of $S U(N)$ and $U(N)$ lattice chiral models.

with $N$ up to $N=200$. Similar results are obtained for $\beta=0.32$. We have also verified that the reduced two-point function does not exponentially decrease at large distance, explaining the behavior of its space integral $\chi$. Finally, note that the results for $S_{1}$ and $S_{2}$, see Table 2, are always consistent with zero, so that they do not give any indication of breaking of the symmetries (2.14) and (2.15).

Our results apparently contradict earlier numerical works [20, 21], which were based on a much smaller statistic, and where the comparison was essentially restricted to the internal energy and the quantity $S_{1}$.

\section{Conclusions}

We have investigated the application of the twisted Eguchi-Kawai reduction procedure to two-dimensional principal chiral models on the lattice, as general testcase of the TEK reduction procedure.

In the strong coupling region, i.e. for $\beta<\beta_{c}$, the large- $N$ limit of the reduced theory appears to reproduce the results available for the original field theory in the large- $N$ limit, and that the TEK reduced model approaches the large- $N$ limit with power corrections of $N^{-2}$. On the other hand, the validity of the reduction prescription apparently breaks down for $\beta>\beta_{c}$. In particular, it is not supported 


\begin{tabular}{cccccc}
\hline \hline$\beta$ & $N$ & $E$ & $\chi$ & $S_{1}$ & $\operatorname{Re} S_{2}$ \\
\hline 0.31 & 10 & $0.5251(1)$ & $22.06(2)$ & $-0.0011(8)+i 0.0004(9)$ & $0.00002(3)$ \\
& 16 & $0.5329(2)$ & $35.46(6)$ & $-0.001(2)+i 0.001(2)$ & $0.00000(3)$ \\
& 20 & $0.5342(2)$ & $43.9(2)$ & $-0.004(3)+i 0.005(3)$ & $-0.00001(5)$ \\
& 30 & $0.5337(2)$ & $64.0(2)$ & $0.003(2)-i 0.005(2)$ & $-0.00002(3)$ \\
& 40 & $0.5317(3)$ & $83.3(6)$ & $0.001(4)+i 0.005(3)$ & $0.00006(4)$ \\
& 60 & $0.5265(3)$ & $123(2)$ & $-0.003(8)+i 0.003(6)$ & $0.00011(5)$ \\
& 80 & $0.5236(2)$ & $157(2)$ & $0.007(6)-i 0.001(5)$ & $0.00003(4)$ \\
& 100 & $0.5216(2)$ & $188(3)$ & $-0.013(9)-i 0.014(8)$ & $0.00005(5)$ \\
& 150 & $0.5204(1)$ & $211(10)$ & $-0.011(7)+i 0.004(10)$ & $0.00002(6)$ \\
& 200 & $0.5200(1)$ & $223(4)$ & $-0.010(9)+i 0.004(9)$ & $0.00004(4)$ \\
\hline 0.32 & 40 & $0.4902(1)$ & $148.2(7)$ & $-0.009(6)-i 0.002(5)$ & $0.00001(4)$ \\
& 60 & $0.4880(2)$ & $235(4)$ & $0.03(2)+i 0.03(2)$ & $-0.00003(7)$ \\
& 80 & $0.4869(2)$ & $331(3)$ & $-0.01(2)-i 0.03(2)$ & $-0.00011(7)$ \\
& 100 & $0.48656(4)$ & $404(2)$ & $0.03(2)-i 0.02(2)$ & $0.00001(4)$ \\
& 150 & $0.48629(8)$ & $565(7)$ & $0.01(2)-i 0.06(2)$ & $0.00013(8)$ \\
\hline \hline
\end{tabular}

Table 2: We report the intenal energy $E$, the magnetic susceptibility $\chi$, and the quantities $S_{1}$ and $S_{2}$, defined in Eqs. (3.3) and (3.4), for $\beta=0.31,0.32$ and several values of $N$.

by the $\mathrm{MC}$ results for the magnetic susceptibility, that do not show evidence of convergence up to $N=200$ and for $\beta$ values corresponding to large- $N$ correlation lengths $\xi \approx 4$ and $\xi \approx 6$ (estimated extrapolating MC data for lattice chiral models). Of course, we cannot exclude that the correct asymptotic regime is eventually set for larger values of $N, N \gg 10^{2}$ say. Although we note that already for smaller values of $\beta \lesssim \beta_{c}$ the approach to the large- $N$ limit is rather slow, in order to explain our results we should suppose a strong slowing down of the convergence with increasing $\beta$. In this regard, we should also add that the equivalence of the theories in the continuum limit requires a uniform convergence to $N=\infty$ when approaching the continuum limit. Another possibility is that our numerical simulations have been trapped by nontrivial extrema of the action, which has nothing to do with the correct limit related to the chiral field theory. However, our numerical checks definitely exclude it at least for $N \lesssim 60$.

We have also checked for possible spontaneous breakings of the symmetries (2.14) and (2.15) of the TEK reduced model, by computing the quantities $S_{1}$ and $S_{2}$, cf. Eqs. (3.3) and (3.4), which are respectively not invariant with respect to the symmetry (2.14) and (2.15). Their expectation values appear to vanish, thus suggesting that the symmetries of TEK are not spontaneously broken, although one cannot exclude breaking of symmetries associated with more complicated order parameters. We recall that one of such symmetries, i.e. the symmetry (2.15), is essential for the demostration of the equivalence of the $N=\infty$ Schwinger-Dyson equations. As- 


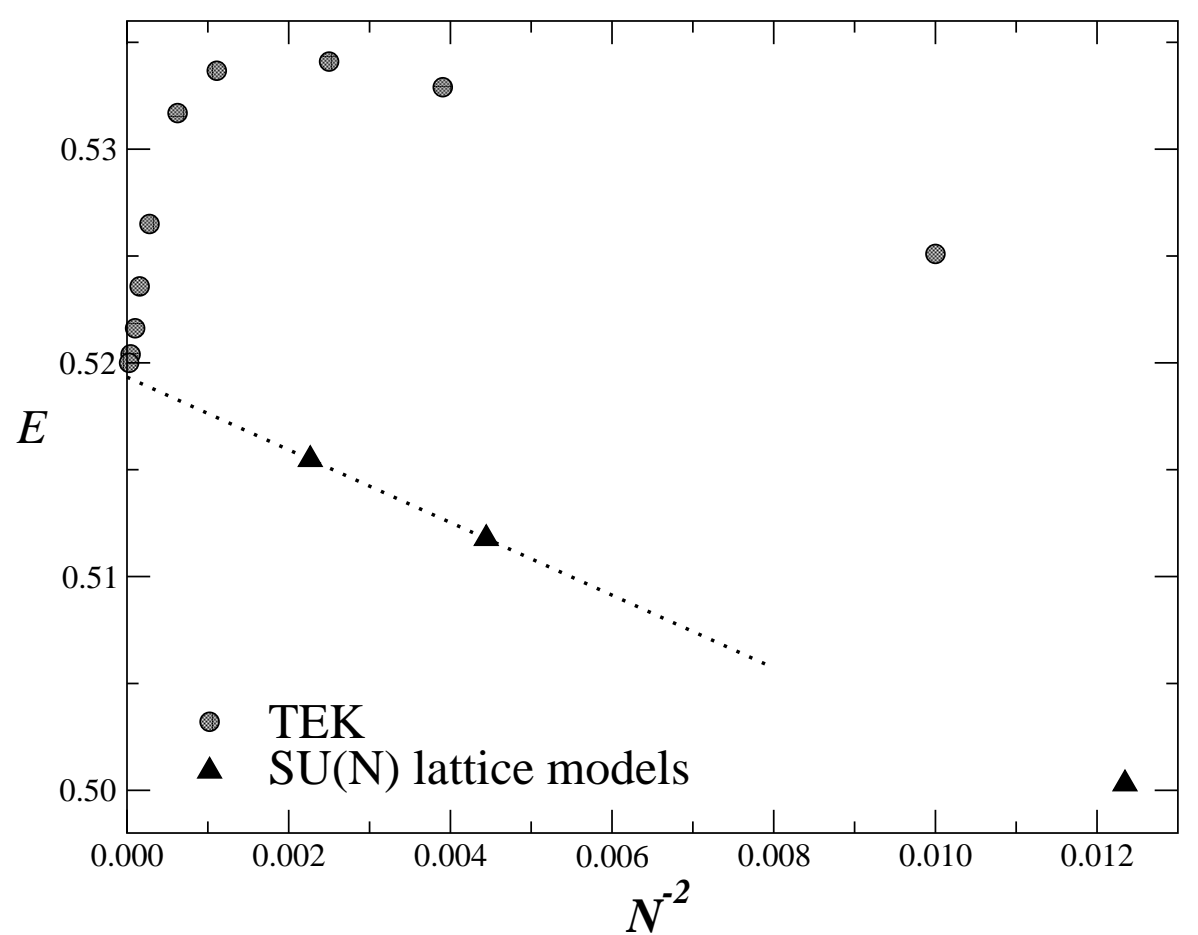

Figure 3: The internal energy versus $N^{-2}$ for $\beta=0.31$. We also show the MC data for $S U(N)$ lattice chiral models, and their large- $N$ extrapolation.

suming that the Schwinger-Dyson equations become formally equal for $N=\infty$, the question to be addressed is whether this is sufficient to identify a theory. Our numerical results suggest that this could not be the case, under the hypothesis that one can recover the $N=\infty$ theory from the finite $N$ behaviour of the reduced theory in its $N \rightarrow \infty$ limit. In other words, the $N=\infty$ TEK reduced theory may be still equivalent to the large- $N$ limit of lattice models, but the limit is singular, so that it cannot be reached from finite values of $N$. This issue is of relevance in any application of the TEK reduction procedure, as in the case of the lattice gauge theory.

The apparent failure of the TEK reduced theory to reproduce the planar limit of lattice chiral models does not necessarily prejudice the possibility of considering the TEK theory system in a different limit, possibly relevant to the fields where twisted Eguchi-Kawai reduced models were recently applied: noncommutative field theories and superstring matrix models. Indeed, TEK reduced chiral models with symmetry group $U(N)$ are formally equivalent to a $\mathrm{U}(1)$ noncommutative theory 26]. This equivalence requires a nontrivial continuum limit that is different from the planar limit we have considered in this work, since one needs to keep the noncommutativity parameter $\theta_{\mu \nu}$ fixed, and $\theta_{\mu \nu}$ scales as $a^{2} N$ where $a$ is the lattice spacing. Thus, the relevant continuum limit should be realized for $a \rightarrow 0$ and $N \rightarrow \infty$ keeping $a^{2} N$ fixed. The lattice spacing is somehow controlled by the coupling $\beta$, which should be appropriately tuned to realize the limit $a \rightarrow 0$. The relation between $a$ and $\beta$ is 


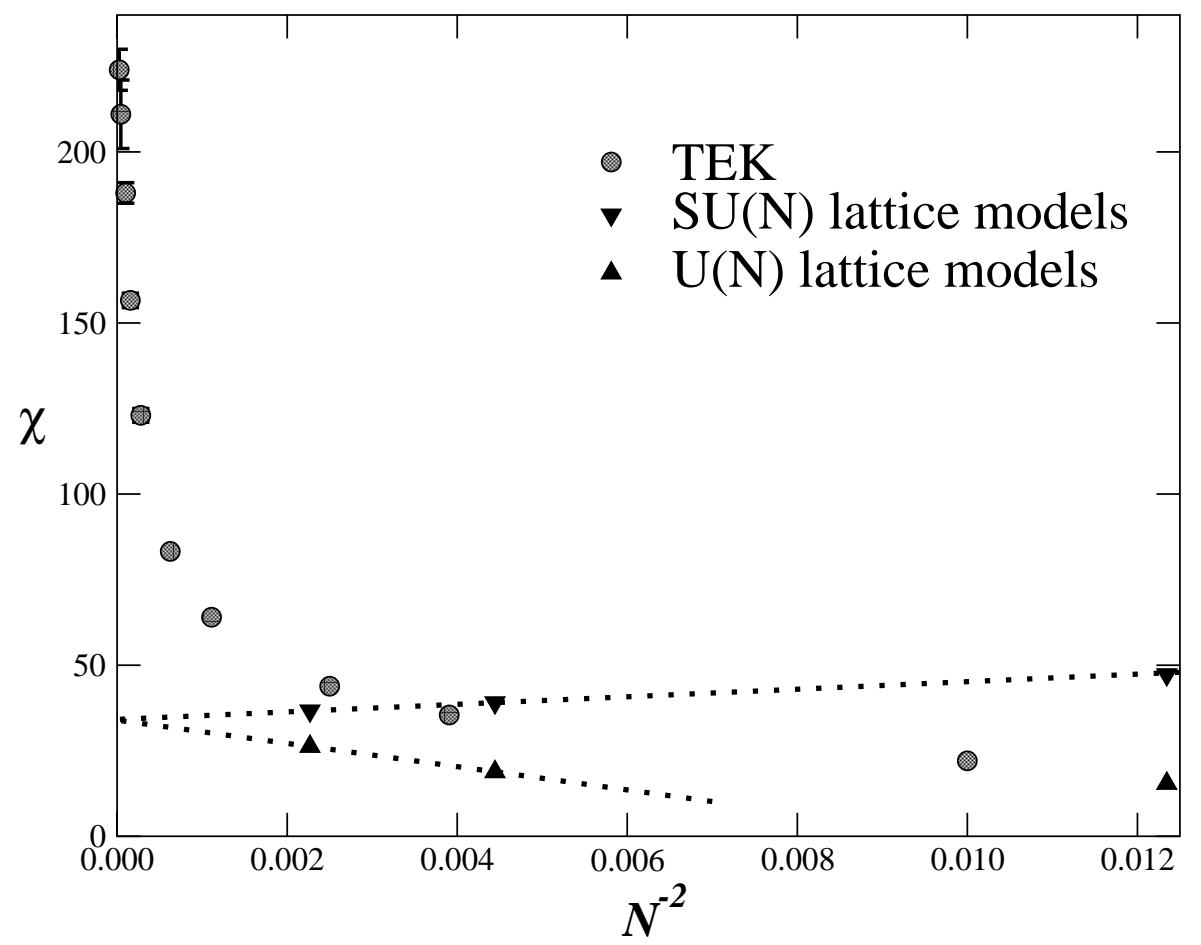

Figure 4: The reduced magnetic susceptibility for $\beta=0.31$ versus $N^{-2}$. We also show the MC data obtained by simulating $S U(N)$ and $U(N)$ lattice chiral models; the lines represent their large- $N$ extrapolations.

therefore essential to define the continuum limit associated with the noncommutative field theory. However, the apparent failure of TEK model to reproduce the planar limit of the principal chiral theory does not allow us to use the known asymptotic behavior of the commutative theory in the continuum limit, for which asymptotic freedom and renormalization group imply $a \sim \exp (-8 \pi \beta)$ in the continuum limit $a \rightarrow 0$ and for $N=\infty$. Of course, this point deserves further investigation.

\section{Acknowledgments}

We thank Luigi Del Debbio, Andrea Pelissetto, and Paolo Rossi for useful discussions. 


\section{References}

[1] T. Banks, W. Fischler, S. H. Shenker, and L. Susskind, Phys. Rev. D 55 (1997) 5112.

[2] N. Ishibashi, H. Kawai, Y. Kitazawa and A. Tsuchiya, Nucl. Phys. B 498 (1997) 467.

[3] Nakajiama and J. Nishimura, Nucl. Phys. B 528 (1998) 355.

[4] H. Aoki, N. Ishibashi, S. Iso, H. Kawai, Y. Kitazawa and T. Tada, Nucl. Phys. B $565(2000) 176$.

[5] H. Aoki, S. Iso, H. Kawai, and Y. Kitazawa, Nucl. Phys. B 573 (2000) 573.

[6] J. Ambjørn, Y.M. Makeenko, J. Nishimura and R.J. Szabo, J. High Energy Phys. 11 (1999) 029; Phys. Lett. B 480 (2000) 399; J. High Energy Phys. 05 (2000) 023; J. High Energy Phys. 07 (2000) 013.

[7] J. Ambjørn, K.N. Anagnostopoulos, W. Bietenholz, T. Hotta, and J. Nishimura, J. High Energy Phys. 07 (2000) 011.

[8] Y. M. Makeenko, Sov. Phys. JETP Lett. 72 (2000) 393.

[9] R. J. Szabo, Quantum field theory on noncommutative spaces, hep-th/0109162.

[10] S. R. Das, Rev. Mod. Phys. 59 (1987) 235.

[11] T. Eguchi and H. Kawai, Phys. Rev. Lett. 48 (1982) 1063.

[12] G. Bhanot, U. M. Heller, and H. Neuberger, Phys. Lett. B 115 (1982) 237.

[13] T. Eguchi and R. Nakayama, Phys. Lett. B 122 (1983) 59.

[14] A. Gonzales-Arroyo and M. Okawa, Phys. Lett. B 133 (1983) 415.

[15] A. Gonzales-Arroyo and M. Okawa, Phys. Rev. D 27 (1983) 2397.

[16] A. Gonzales-Arroyo and C. P. Korthals Altes, Phys. Lett. B 131 (1983) 336.

[17] K. Fabricius and O. Haan, Phys. Lett. B 143 (1984) 459.

[18] O. Haan and K. Meier, Phys. Lett. B 175 (1986) 192.

[19] B. Aneva, Y. Brihaye and P. Rossi, Phys. Lett. B 134 (1984) 245.

[20] S. R. Das and J. B. Kogut, Nucl. Phys. B 235 (1984) 521.

[21] A. Gonzales-Arroyo and M. Okawa, Nucl. Phys. B 247 (1984) 104.

[22] F. Green and S. Samuel, Nucl. Phys. B 190 (1981) 113.

[23] A. M. Polyakov, Gauge Fields and Strings, Harwood Academic Publishers, New York 1988. 
[24] P. Rossi, M. Campostrini, and E. Vicari, Phys. Rept. 302 (1998) 143.

[25] L. Del Debbio, H. Panagopoulos, P. Rossi, and E. Vicari, Phys. Rev. D 65 (2002) 021501; J. High Energy Phys. 01 (2002) 009.

[26] S. Profumo, Noncommutative Principal Chiral Models, hep-th/0111285.

[27] F. Green and S. Samuel, Phys. Lett. B 103 (1984) 110.

[28] P. Rossi and E. Vicari, Phys. Rev. D 49 (1994) 1621; Phys. Rev. D 49 (1994) 6072; Phys. Rev. D 55 (1997) 1698 (E).

[29] M. Campostrini, P. Rossi and E. Vicari, Phys. Rev. D 51 (1995) 958; Phys. Rev. D 52 (1995) 358; Phys. Rev. D 52 (1995) 386.

[30] M. Campostrini, P. Rossi and E. Vicari, Phys. Rev. D 52 (1995) 395.

[31] G.'t Hooft, Comm. Math. Phys. 81 (1981) 529. 